\title{
« Une campagne efficace qui a su conscientiser les jeunes femmes au cancer du sein » : Résultats des campagnes de sensibilisation au cancer du sein auprès des jeunes Canadiennes
}

\author{
par Lorna Larsen
}

\section{RÉSUMÉ}

La présente étude pluriannuelle visait à reproduire le succès d'une campagne de sensibilisation au cancer du sein auprès des jeunes femmes et d'en évaluer l'efficacité. La campagne a été menée dans plusieurs établissements postsecondaires canadiens. Elle a été orchestrée par un organisme caritatif canadien, léquipe Shan de sensibilisation au cancer du sein chez les jeunes femmes (Team Shan Breast Cancer Awareness for Young Women) dans les campus collégiaux et universitaires de l'Ouest canadien entre 2010 et 2016. L'étude était construite sur l'administration de questionnaires distribués à de jeunes femmes (de 17 à 29 ans) sur 11 campus avant $(n=880)$ et après $(n=794)$ la campagne de sensibilisation. Les questions évaluaient les activités de sensibilisation, les principaux messages à retenir et le transfert de connaissances sur le cancer du sein. L'équipe Shan a élaboré différentes stratégies pour sensibiliser les jeunes femmes au risque de développer un cancer du sein. Les campagnes se sont révelées un outil de santé publique efficace et ont eu une incidence positive sur les jeunes femmes. Les résultats de létude suggèrent que les stratégies de promotion de la santé sont utiles pour informer les jeunes femmes, un groupe à risque de souffrir d'un cancer du sein.

Mots-clés : cancer du sein, sensibilisation, jeunes femmes, diagnostic tardif, détection précoce, promotion de la santé

\section{CONTEXTE}

$\mathrm{O}$ n prévoit qu'environ 8600 adolescentes et jeunes adultes de 15 à 39 ans recevront chaque année un diagnostic de cancer du sein au Canada (Canadian Cancer Society/Société canadienne du cancer, 2019). Le chemin qui les attend est particulièrement épineux et les besoins qui ne trouveront pas réponse peuvent nuire au pronostic et au traitement, en plus d'avoir des conséquences à court et à long terme, ainsi que des répercussions sur leur survie (Canadian Partnership Against

\section{AUTEURE}

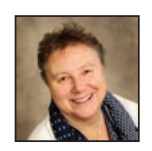

Lorna Larsen, inf. aut., B.Sc.inf., Présidente de l'équipe Shan, Équipe Shan pour la sensibilisation au cancer du sein chez les jeunes femmes (équipe Shan), 18 Beechwood Path, Huntsville (Ontario) Canada P1H 1S8

519-535-1503

Profil ORCiD : https://orcid.org/0000/0002/3230/686X

Irlarsen@sympatico.ca

DOI: $10.5737 / 236880763216874$
Cancer/Partenariat canadien contre le cancer, 2017). Les adolescentes et jeunes adultes sont plus à risque de recevoir un diagnostic tardif; il y a donc des lacunes dans la détection précoce pour ce groupe (Ramphal, 2016). Ces lacunes, attribuables à l'inaction du médecin ou de la patiente, peuvent entraîner des conséquences néfastes chez les jeunes femmes qui reçoivent tardivement leur diagnostic et leur causer une détresse profonde (Miles, 2017). Pour encourager à agir les populations d'adolescentes et de jeunes adultes en bonne santé, dans le cadre de la présente étude, des campagnes de sensibilisation au cancer et au dépistage des symptômes ont donc été réalisées pour encourager les adolescentes et les jeunes adultes en bonne santé à agir (Kyle, 2012).

$\mathrm{Au}$ Canada, le cancer du sein est le plus fréquent chez les femmes de 15 à 39 ans (Canadian Partnership Against Cancer/Partenariat canadien contre le cancer, 2019). Son incidence chez les jeunes Canadiennes fluctue, mais elle augmente considérablement avec l'âge. Selon les recherches, il y a de plus en plus de jeunes femmes (âgées de 15 à 39 ans) qui présentent un cancer du sein métastatique et dont l'issue de la maladie est défavorable, notamment à cause du taux de survie moins élevé (Johnson et al., 2018). Qui plus est, cette population est plus susceptible que les autres de vivre la détresse en recevant le diagnostic (Miroshnychenko, 2021).

Certaines recherches recommandent des stratégies de communication pour combler les lacunes dans la sensibilisation des jeunes femmes au cancer du sein. En informant les jeunes femmes sur les risques de cancer du sein, les symptômes et l'autodétection, il est possible de leur inculquer à vie des comportements qui les aideront à réduire les risques, à détecter le cancer au stade précoce et à promouvoir la santé et le bienêtre global. C'est pour répondre à ce besoin qu'émergent de nouvelles approches personnalisées de promotion de la santé ciblant les jeunes femmes (ex. Bottorff et al., 2014).

Léquipe Shan (Team Shan Breast Cancer Awareness for Young Women), un organisme caritatif canadien enregistré, travaille à sensibiliser les jeunes femmes à la détection précoce, à la réduction des risques et à la prévention du cancer du sein. L'équipe a été nommée ainsi en l'honneur de Shanna (Shan) Larsen, une jeune femme qui a reçu diagnostic erroné après plusieurs consultations médicales et qui a fini par apprendre qu'elle souffrait d'un cancer du sein métastatique à un stade avancé. Shan a été emportée par la maladie alors qu'elle n'avait que 24 ans. 


\section{Premières initiatives}

En 2006, l'équipe Shan a commandé une revue de littérature pour clarifier les besoins informationnels des jeunes femmes au sujet du cancer du sein et pour analyser les stratégies de promotion de la santé s'attaquant aux lacunes de sensibilisation et d'éducation. L'exercice a révélé qu'il serait possible de réduire le nombre de décès attribuables au cancer du sein dans cette tranche d'âge en favorisant la détection précoce. Il n'en reste pas moins que, pour y arriver, il faut enseigner aux jeunes femmes les signes et les symptômes du cancer du sein afin qu'elles soient en mesure de le détecter. On peut traiter le cancer du sein, et les campagnes d'éducation, lorsqu'elles sont efficaces, permettent de réduire le nombre de décès (Suffel et Coleman, 2006).

En se fondant sur les conclusions de sa revue de littérature, l'équipe Shan a préparé une affiche et une brochure donnant des informations sur le cancer du sein, les symptômes, les facteurs de risque et l'autogestion (détection précoce et réduction des risques). Sur les documents imprimés figuraient aussi une photo de Shan et un mot sur son histoire. Ces outils ont été testés en 2006 auprès de 293 jeunes femmes de 15 à 29 ans dans six sites communautaires ciblés du centre du pays. Les résultats ont été concluants et ont motivé la diffusion à grande échelle du matériel éducatif élaboré par l'équipe Shan. Les documents s'adressaient aux jeunes femmes et venaient combler des lacunes importantes au niveau des connaissances. Règle générale, les commentaires forts positifs témoignaient d'une curiosité pour le sujet et d'un désir de l'approfondir. Plus important encore, les jeunes femmes ont compris que le risque de cancer était bien réel, peu importe leur âge (Larsen et Forchuk, 2006).

En 2007, à partir des résultats du groupe test, l'équipe Shan a créé et orchestré, dans le centre du pays, une campagne de sensibilisation aux multiples facettes du cancer du sein. Les objectifs à court terme de ce projet pilote étaient : 1) la mise sur pied d'une vaste campagne de sensibilisation au cancer du sein ciblant les jeunes femmes; 2) l'évaluation de l'efficacité des stratégies et des documents de ladite campagne; 3) l'accroissement des connaissances des jeunes femmes sur le cancer du sein. Les objectifs à long terme étaient : 1) l'accentuation de la détection précoce du cancer du sein chez les jeunes femmes; 2) l'amélioration de l'état de santé des jeunes femmes diagnostiquées avec un cancer. La campagne avait pour thème « Le cancer du sein... une maladie qui ne touche pas que les femmes âgées » (en anglais : Breast Cancer... not just a disease of older women). Une revue des écrits les plus récents a été commandée pour valider le message de la campagne (Suffel et Coleman, 2007).

La campagne prévoyait des publicités dans les médias et des activités de marketing, la distribution de documents imprimés, des affiches et des présentations, ainsi que la création d'un site Web et de publications pour les médias sociaux. De plus, un questionnaire d'évaluation a été distribué immédiatement avant et après la campagne pilote afin d'en évaluer l'efficacité. Les jeunes femmes de 15 à 29 ans qui ont accepté de répondre au questionnaire avant $(n=293)$ et après $(n=301)$ formaient un échantillon de commodité et provenaient de six sites communautaires ciblés. La majorité des participantes $(86,0 \%)$, tous sites confondus, avaient remarqué les annonces de la campagne. Elles se rappelaient surtout que le cancer du sein frappe à tous les âges sans discrimination (57,0\%). Après la campagne, seulement 3,8 \% des participantes ont dit ne rien savoir sur le cancer du sein comparativement à 10,2\% avant la campagne. Les commentaires reçus étaient majoritairement très positifs et exprimaient généralement des félicitations et de la reconnaissance envers l'équipe et son travail. Somme toute, l'approche multidimensionnelle de l'équipe Shan a su répondre aux besoins informationnels des jeunes femmes sur le cancer du sein et les sensibiliser aux risques. Les résultats positifs de la campagne pilote nous ont donné l'espoir de voir grandir le projet (Larsen et Forchuk, 2008).

\section{BUT DE L'ÉTUDE}

Le présent article fait état d'une étude pluriannuelle visant à reproduire le succès de la campagne pilote de l'équipe Shan menée auprès des jeunes femmes pour les sensibiliser au cancer du sein, et d'en évaluer l'efficacité dans plusieurs établissements postsecondaires canadiens.

\section{PORTÉE DE LA CAMPAGNE}

Léquipe Shan a mené des campagnes de sensibilisation au cancer du sein dans des campus postsecondaires de l'Ouest canadien de 2010 à 2015 pendant les mois d'octobre et novembre de chaque année. Les jeunes femmes étaient sollicitées grâce à l'affichage installé sur les campus et aux demandes formulées directement à l'équipe Shan (ex. exposé devant un club étudiant, conférence-midi du centre des femmes, activité du centre des sports). Les ressources papier (affiches, brochures et signets), les activités réalisées grâce à des partenariats établis sur le campus et le bouche-à-oreille ont aussi permis d'élargir la portée de la campagne. En plus, il a été possible d'interpeller indirectement un vaste auditoire grâce à des publicités dans les endroits publics (transport en commun, abribus, panneau d'affichage) et dans les médias du campus (radio, journaux étudiants, télévision en circuit fermé), ainsi qu'au site Web de l'équipe Shan et aux messages de la campagne publiés dans les médias sociaux (Facebook). Ces messages étaient les mêmes que ceux de la campagne pilote, c'est-à-dire des informations sur le cancer, les symptômes, les facteurs de risque et la stratégie d'autogestion (pour la détection précoce et la réduction des risques) et reprenaient le thème « Le cancer du sein... une maladie qui ne touche pas que les femmes âgées ».

Durant l'étude, deux revues de littérature ont permis de confirmer la validité du message en l'appuyant sur les plus récentes recherches. L'exercice a aussi fait ressortir un certain nombre de recommandations dont a tenu compte l'équipe Shan pour développer et améliorer ses campagnes (Chadder et Coleman, 2011; Symonds, Tiseo et Coleman, 2015).

\section{MÉTHODOLOGIE}

Construite sur un principe d'évaluation a priori et a posteriori, l'étude sondait de jeunes femmes âgées de 17 à 29 ans de 
11 campus ciblés à l'aide d'un questionnaire administré avant et après la tenue de la campagne. Les questions évaluaient les activités de la campagne de sensibilisation, les principaux messages retenus, l'utilisation prévue, par les participantes, de l'information reçue pendant la campagne, le partage des informations apprises pendant la campagne et le niveau autodéclaré de connaissances et de sensibilisation sur le cancer du sein. Comme les questionnaires d'évaluation étaient anonymes, la présente étude n'a pas eu à obtenir l'approbation du comité de révision déontologique (gouvernement du Canada, 2018).

\section{Cadre et participantes}

Léquipe Shan a installé des stations d'évaluation sur les campus dans des zones où circulent beaucoup d'étudiants. Des communications écrites et orales invitaient les étudiantes à se porter volontaires pour évaluer la campagne. Le questionnaire pré-campagne a été administré à un échantillon de commodité de jeunes femmes âgées de 17 à 29 ans sur les campus qui ont accepté d'y répondre sur papier. Pendant le déroulement de la campagne, un deuxième échantillon de commodité constitué lui aussi de jeunes femmes âgées de 17 à 29 ans recrutées sur les campus a accepté de répondre sur papier au questionnaire post-campagne. En répondant aux questionnaires autoadministrés, les femmes donnaient leur consentement implicite à participer à l'étude.

\section{Collecte de données}

Un membre de l'équipe Shan était sur place pour encadrer l'évaluation. Les questionnaires administrés avant et après la campagne ont été rédigés en consultation avec un consultant de recherche indépendant. Les outils contenaient des questions qui concordaient avec les questionnaires d'évaluation de la campagne pilote; ils ont été peaufinés avec le temps, mais n’ont pas été formellement validés. Les réponses au questionnaire pré-campagne ont été reçues avant le lancement de la campagne, qui avait lieu pendant le premier trimestre, tandis que les réponses au questionnaire post-campagne ont été obtenues après la fin de la campagne, qui coïncidait avec la fin de l'année scolaire. Il fallait environ une minute pour répondre au questionnaire pré-campagne et environ cinq minutes pour le questionnaire post-campagne. De 2011 à 2016, les questionnaires post-campagne contenaient des questions ouvertes et des questions fermées. Le tableau I présente la liste complète des questions posées dans les outils d'évaluation de la campagne de l'équipe Shan.

\section{Analyse des données}

Le consultant de recherche de la campagne a compilé les résultats et étudié les données d'évaluation. Les réponses aux questions ouvertes ont été analysées et codées afin de relever les thèmes récurrents. Dans l'analyse, un schéma de codage a été établi pour catégoriser les réponses récurrentes. Ces catégories ont servi à coder les réponses du questionnaire et à résumer les résultats en tableaux. Une analyse fréquentielle a servi à décrire les données démographiques, les questions fermées et les données catégorielles. Les pourcentages ont été arrondis à la première décimale.

\section{RÉSULTATS}

\section{Données démographiques}

Les participantes ( $n=1674$ ) étaient des femmes, âgées de 17 à 29 ans, représentatives de la diversité étudiante des établissements postsecondaires canadiens ainsi que du groupe ciblé par la campagne. L'étude s'est déroulée sur plusieurs années et, pendant cette période, 880 participantes ont répondu au questionnaire pré-campagne et 794 au questionnaire post-campagne.

\section{Publicités de la campagne}

Comme pour la campagne pilote, la majorité $(91,9 \%)$ des répondantes au questionnaire post-campagne $(\mathrm{n}=794)$ de tous les établissements ont effectivement remarqué les publicités de la campagne de l'équipe Shan. Dans chacun des campus sans exception, les activités de la campagne ayant attiré le plus d'attention étaient celles liées aux médias et au marketing (87,8\%), aux documents imprimés $(63,6 \%)$ et aux médias sociaux et interactifs $(22,0 \%)$.

Selon des répondantes $(n=485)$ aux évaluations des campagnes de 2010 à 2012, l'utilisation des médias et le marketing publicitaire constituaient la stratégie de sensibilisation la plus efficace $(60,8 \%)$. D'autres participantes $(n=552)$ avaient surtout remarqué les publicités dans les transports en commun, comme les autobus ou les trains légers (67,4\%), suivies des publicités dans les abribus $(42,9 \%)$ et des panneaux d'affichage $(35,1 \%)$. Du côté des publicités dans les médias, ce sont les publicités dans les journaux étudiants qui ont le plus attiré l'attention (26,3\%). Et du côté des documents imprimés, les participantes avaient remarqué le plus souvent remarqué ( $n=542)$ l'affiche de la campagne $(55,7 \%)$, la brochure $(31,7 \%)$ et le signet $(10,9 \%)$.

La présence dans les médias interactifs et les médias sociaux la plus remarquée était les publications sur la page Facebook de l'équipe Shan (11,2 \%) suivies de celles sur d'autres sites Internet (3,6 \%), dont ceux des partenaires universitaires et collégiaux de la campagne, et le site Web de l'équipe Shan (3,0\%). Google Analytics a fourni des détails sur la fréquentation des sites Web. Les jeunes femmes des établissements d'enseignement ciblés par la campagne ont souvent visité la page de l'histoire de Shan et la page intitulée « Breast Aware » sur le site Web teamshan.ca. Les interactions sur la page Facebook ont aussi été colligées. De nouveaux internautes se sont abonnés à la page Facebook et les photos marketing de la campagne ont reçu des « j'aime ». Les vidéos (ex. Check Yourself Canada) et les articles de blogue de l'équipe Shan ont également été consultés via des liens sur Facebook.

\section{Messages à retenir et mesures à prendre}

Comme dans le cas de la campagne pilote, le message le plus marquant pour les participantes ayant remarqué la campagne $(n=638)$ est le fait que le cancer du sein frappe à tous les âges sans discrimination (58,3\%). L'importance des mesures d'autogestion (autosoins) comme l'auto-examen des seins arrive en deuxième position (20,7 \%). Le tableau 2 donne la liste complète des thèmes retenus et des résultats. 
Tableau 1

Questions d'évaluation de la campagne de sensibilisation au cancer du sein de l'équipe Shan

Évaluation des activités de la campagne (campagnes de 2010 à 2012)

1. Avez-vous vu ou entendu les publicités médias et marketing de l'équipe Shan sur le cancer du sein chez les jeunes femmes? Cochez tous les éléments qui s'appliquent.

a) Journal étudiant

b) Train léger/autobus

c) Gare de train léger/abribus

d) Panneau d'affichage

e) Radio

f) Autre. Veuillez préciser :

g) Aucune de ces réponses

2. Avez-vous vu les documents imprimés suivants produits pour la campagne de sensibilisation au cancer du sein de l'équipe? Cochez tous les éléments qui s'appliquent.

a) Brochure

b) Affiche

c) Signet

d) Autre. Veuillez préciser :

e) Aucune de ces réponses

3. Avez-vous vu les publications de l'équipe Shan en ligne ou sur les médias sociaux? Cochez tous les éléments qui s'appliquent.

a) Site Web de l'équipe Shan

b) Page Facebook de l'équipe Shan pour la sensibilisation des jeunes femmes au cancer du sein

c) Autres sites Internet

d) Aucune de ces réponses

Évaluation de l'efficacité des activités de la campagne (campagnes de 2010 à 2012)

4. Quelle stratégie de sensibilisation vous a le plus conscientisée? Cochez une seule réponse.

a) Publicités médias et marketing

b) Documents imprimés

c) Médias sociaux et médias interactifs

Évaluation des activités de la campagne (campagnes de 2013 à 2015)

5. Avez-vous vu ou entendu les publicités suivantes de la campagne sur le cancer du sein chez les jeunes femmes de l'équipe Shan? Cochez tous les éléments qui s'appliquent.

a) Marketing (autobus, abribus, panneaux d'affichage)

b) Médias (radio universitaire, journaux)

c) Documents imprimés (affiches, brochures, signets)

d) Médias sociaux et interactifs (site Web, Facebook)

e) Autre. Veuillez préciser :

f) Aucune de ces réponses

Évaluation des messages à retenir (cde 2010 à 2012)

6. Quel est le message principal que vous retenez des publicités médias et marketing?

7. Quel est le message principal que vous retenez des documents imprimés?

Évaluation des messages à retenir (campagnes de 2013 à 2015)

8. Quel est le message principal que vous retenez des publicités de la campagne de l'équipe Shan?

Évaluation de l'utilisation prévue de l'information reçue (campagnes de 2013 à 2015)

9. Quels gestes poserez-vous après avoir vu ou entendu les publicités de la campagne de l'équipe Shan?

Évaluation de la fréquence du partage d'information sur la campagne (campagnes de 2013 à 2015)

10. À combien de personnes avez-vous parlé de ce que vous avez appris grâce à la campagne de l'équipe Shan?

a) 0

b) $1-2$

c) $3-5$

d) $6-10$

e) Plus de 10

Évaluation du niveau de connaissance sur le cancer du sein (campagnes de 2010 à 2013)

11. Comment évaluez-vous votre niveau actuel de connaissance sur le cancer du sein chez les jeunes femmes?

a) Aucune connaissance

b) Certaines connaissances

c) Bien informée

Évaluation du niveau de sensibilisation au cancer du sein (campagnes 2010-2013)

12. Que savez-vous présentement sur le cancer du sein?

a) Informations sur le cancer du sein

b) Symptômes

c) Autogestion

d) Facteurs de risque

Observations générales (campagnes de 2010 à 2015)

13. Formulez ici tout autre commentaire pertinent sur la campagne de sensibilisation de l'équipe Shan au cancer du sein chez les jeunes femmes. 
Tableau 2

Thèmes des principaux messages à retenir (campagnes de 2010 à 2015)

\begin{tabular}{|c|c|}
\hline Thèmes & $\begin{array}{c}\text { Tous les } \\
\text { établissements } \\
(n=638)\end{array}$ \\
\hline $\begin{array}{l}\text { Le cancer du sein frappe à tous les âges sans } \\
\text { discrimination (femmes jeunes ou âgées) }\end{array}$ & $372(58,3 \%)$ \\
\hline $\begin{array}{l}\text { Messages concernant l'autosoin (examen } \\
\text { mammaire, détection précoce) }\end{array}$ & $132(20,7 \%)$ \\
\hline $\begin{array}{l}\text { Sensibilisation au cancer du sein (maladie } \\
\text { grave) }\end{array}$ & $113(17,7 \%)$ \\
\hline $\begin{array}{l}\text { Servir la cause (appuyer un traitement, faire } \\
\text { un don) }\end{array}$ & $44(6,9 \%)$ \\
\hline Histoire de Shan (décès tragique) & $37(5,8 \%)$ \\
\hline Campagne positive (efficace, instructive) & $33(5,2 \%)$ \\
\hline Incidence (prévalence) & $6(0,9 \%)$ \\
\hline
\end{tabular}

${ }^{*}$ Certaines participantes ont coché plus d'une réponse. Toutes les réponses figurent au tableau.

La majorité $(93,3 \%)$ des participantes $(\mathrm{n}=208)$ aux campagnes de 2013 et 2015 ont dit avoir commencé à suivre les conseils de l'équipe Shan ou prévoir le faire. Le plus souvent, elles comptaient procéder à un l'auto-examen des seins ou faire un examen médical (41,3\%). Le tableau 3 donne la liste complète des résultats et des actions personnelles (70,2 \%), interpersonnelles $(26,0 \%)$ et sociétales $(16,3 \%)$. La majorité $(54,8 \%)$ des participantes $(n=219)$ aux campagnes de 2013 et 2015 ont dit ne pas avoir communiqué d'information sur la campagne à autrui, alors que 45,2 \% en ont parlé à au moins une personne.

\section{Niveaux de connaissance sur le cancer du sein}

D'après les réponses aux questionnaires pré-campagne de 2010 à 2013, la majorité $(76,6 \%)$ des participantes $(n=880)$ possédaient «certaines connaissances » sur le cancer du sein. Dans les questionnaires post-campagne, cette proportion s'élevait à 78,4 \% $(n=685)$. Dans les réponses post-campagne, on observait aussi une hausse (+4,9\%) du nombre des participantes qui se disent «bien informées » sur le cancer $\mathrm{du}$ sein, ainsi qu'une baisse (-6,6\%) du nombre de participantes jugeant n'avoir « aucune connaissance » à ce sujet. Les répondantes aux questionnaires administrés avant $(n=853)$ et après $(n=663)$ la campagne ont surtout indiqué bien connaître les faits sur le cancer du sein, les symptômes et les facteurs de risque. On a constaté au fil du temps une augmentation constante du nombre de participantes se disant sensibilisées à ces aspects $(+11,6 \%,+4,3 \%$ et $+8,4 \%$ respectivement). L'autogestion (détection précoce et réduction des risques) est le thème que les participantes connaissaient le moins bien. Avec le temps, on a également observé une augmentation (+7,5\%) de la sensibilisation à l'autogestion.
Tableau 3

Thèmes des actions entreprises ou à réaliser (campagnes de 2013 à 2015)

\begin{tabular}{lc}
\hline \multicolumn{1}{c}{ Thèmes } & $\begin{array}{c}\text { Tous les } \\
\text { établissements } \\
(\mathbf{n}=208)\end{array}$ \\
\hline Action personnelle & \\
\hline $\begin{array}{l}\text { Examen mammaire (auto-examen, examen } \\
\text { médical) }\end{array}$ & $86(41,3 \%)$ \\
$\begin{array}{l}\text { Mieux s'informer sur le cancer du sein } \\
\text { (facteurs de risque, symptômes) }\end{array}$ & $34(16,3 \%)$ \\
Se renseigner sur la campagne de l'équipe & $17(8,2 \%)$ \\
Shan & \\
Prendre soin de soi (mode de vie sain) & $9(4,3 \%)$ \\
Action interpersonnelle & \\
\hline En parler à ses proches & $45(21,6 \%)$ \\
Les encourager à se faire examiner & $9(4,3 \%)$ \\
Action sociétale & $8(3,8 \%)$ \\
\hline Soutenir la cause & $6(2,9 \%)$ \\
\hline Incertaine des actions à poser & $34(16,3 \%)$ \\
\hline Aucune action entreprise & \\
\hline Certaines & \\
\hline
\end{tabular}

*Certaines participantes ont coché plus d'une réponse. Toutes les réponses figurent au tableau.

\section{Commentaires sur la campagne}

Comme pour la campagne pilote, les réponses $(\mathrm{n}=250)$ aux questions ouvertes permettant de formuler des commentaires étaient très positives et soulignaient l'importance de la campagne (ex. «Quelle bonne chose que de sensibiliser les femmes de tout âge au cancer du sein. (;) »). À maintes reprises, les participantes ont remercié les bénévoles de l'équipe Shan d'avoir été sur le campus pour sensibiliser les jeunes femmes (ex. « Merci de nous avoir transmis ces informations cruciales. Continuez votre bon travail! »). Les participantes ont également bien accueilli l'histoire de Shan pour sensibiliser les jeunes femmes (ex. « Très bonne campagne. L'histoire vraie de cette jolie jeune femme rend la maladie tellement plus réelle et nous force à la prendre au sérieux. »). Quelques participantes ont suggéré des stratégies de campagne, qui donneraient, selon elles, des résultats efficaces. D'autres ont plutôt commenté les connaissances acquises (ex. « La campagne sensibilise les jeunes femmes comme moi aux risques qu'elles courent. »). Les jeunes femmes n'avaient aucun commentaire négatif à formuler sur les campagnes et en redemandaient toujours davantage (ex. « Poursuivez votre bon travail de sensibilisation. »). 


\section{DISCUSSION}

Dans ses campagnes de sensibilisation au cancer du sein, l'équipe Shan a mis en œuvre diverses stratégies pour informer les jeunes femmes de leur risque de développer le cancer $\mathrm{du}$ sein. La grande majorité des jeunes femmes ont dit avoir vu les campagnes et leur réaction était positive. On peut donc en déduire que les campagnes ont bien atteint la population cible. Règle générale, les publicités marketing ont été les plus vues. Les documents imprimés se sont également avérés efficaces. Peu de participantes ont pris connaissance de la campagne sur les médias sociaux, même si on nous avait recommandé d'y être présents. Possible cause : la consultation des médias interactifs et des médias sociaux repose sur une décision active, alors que la publicité diffusée sur les campus est passive. Probablement que des personnes non sondées ont consulté les médias sociaux, dont l'utilisation augmente au fil du temps, si bien qu'on encourage à continuer sur cette voie. Dans l'ensemble, les résultats étaient prometteurs et indiquent que les jeunes femmes ont remarqué différentes formes de publicités.

Le message le mieux retenu par les participantes (et l'un des principaux messages que voulait transmettre l'équipe Shan) est que le cancer du sein frappe quel que soit l'âge. D'après les résultats aux questionnaires post-campagne, les jeunes femmes entendaient prendre des mesures à différents niveaux (personnels, interpersonnels et sociétaux). Selon les résultats, une forte proportion des participantes n'avait pas partagé l'information de la campagne avec autrui, mais elles étaient nombreuses à vouloir le faire. Peut-être n'avaient-elles pas eu l'occasion de le faire avant l'évaluation post-campagne, mais elles ont manifesté leur intention de le faire dans l'avenir (ex. «Cette campagne est essentielle; vous pouvez compter sur moi pour passer le mot. :) ». Les résultats sont encourageants; ils montrent que certaines participantes ont rapidement disséminé l'information et que d'autres comptaient le faire.

La majorité des répondantes aux questionnaires ayant suivi les campagnes de 2011 à 2014 ont dit avoir « certaines connaissances » sur le cancer du sein. La proportion de jeunes femmes n'ayant « aucune connaissance » a diminué alors que celle des jeunes femmes «bien informées » a augmenté après les campagnes, ce qui témoigne du succès des campagnes de sensibilisation. Comparativement aux résultats obtenus avant la campagne, les répondantes aux questionnaires post-campagne rapportaient systématiquement de meilleures connaissances au sujet du cancer du sein, des symptômes et des facteurs de risque. De manière générale, la sensibilisation à l'autogestion a aussi augmenté, mais les résultats font ressortir le besoin de donner davantage d'information sur les autosoins lors des prochaines campagnes.

L'histoire vraie de Shanna a capté l'attention de la population ciblée et permis de mieux faire passer le message. En effet, l'histoire de Shan est marquante et prouve que le cancer du sein frappe à tous les âges (ex. « La campagne m’a touché parce Shan avait mon âge. »). L'équipe Shan a su utiliser à bon escient l'histoire de Shan et le point de vue des jeunes femmes pour accroître la sensibilisation au cancer du sein. Les jeunes femmes ont suggéré d'autres moyens de leur transmettre l'information dans les campagnes à venir, et l'équipe Shan a décidé d'adapter ses activités et son message en conséquence. En outre, le fait de mettre de l'avant des stratégies d'autogestion et de prévention dans les publicités, puis de demander aux jeunes femmes d'évaluer les ressources de l'équipe Shan leur permet de gagner en confiance et augmente les chances qu'elles passent à l'action (ex. " Les annonces publicitaires menaient au site Web. La campagne m’a touchée. »; « Très bonne campagne, c'est important de sensibiliser les jeunes femmes. J'en sais peu à ce sujet, mais je vais m'informer. »; « A force d'apercevoir les panneaux et les affiches, j'ai fini par télécharger l'application pour me rappeler de faire un examen mammaire. »)

En général, les commentaires sur la campagne étaient très positifs et montraient que les participantes avaient apprécié la campagne et l'information transmise. Même qu'elles en redemandaient. Pendant la durée de l'étude, les stratégies de sensibilisation ont légèrement évolué en fonction des commentaires des jeunes femmes sur les campus et de la recension des recherches les plus récentes. Dans l'ensemble, les résultats des campagnes pluriannuelles étaient néanmoins en phase avec ceux de la campagne pilote.

\section{Limites de l'étude}

L'étude n'avait pas de groupe témoin et avait recours à un échantillonnage consécutif de commodité pour évaluer la campagne. D'autres études seront nécessaires pour savoir s'il y a effectivement à long terme une réduction des risques liés au cancer du sein et une modification des comportements d'autodétection chez les jeunes femmes. De plus, il pourrait s'avérer utile d'étudier plus en détail l'incidence des messages diffusés sur les médias sociaux selon l'âge des participantes et le statut d'étudiante.

\section{Recommandations}

Il est recommandé de continuer à mettre en œuvre les stratégies efficaces mises au point par l'équipe Shan pour promouvoir la santé auprès des jeunes femmes sur les campus, d'intégrer les suggestions des jeunes femmes aux futures campagnes, d'actualiser le contenu de la campagne à partir des données probantes et d'accroitre le rayonnement de la campagne en profitant des possibilités sur les campus, dans les collectivités et en ligne.

\section{IMPLICATIONS POUR LES SOINS INFIRMIERS}

Nombreuses sont les façons de répondre aux besoins en matière de sensibilisation et d'éducation des adolescents et des jeunes adultes canadiens en bonne santé, que ce soit au niveau de la gestion, de la pratique et de la recherche en soins infirmiers. Il existe des programmes pratiques de soins infirmiers pour faciliter la promotion de la santé auprès des adolescents et des jeunes adultes et d'ainsi leur transmettre de l'information cruciale sur le cancer en faisant appel aux initiatives de santé publique en place (ex. santé sexuelle, santé familiale, santé scolaire et prévention des maladies chroniques), de même qu'aux services de santé pour les étudiants postsecondaires, aux cliniques de santé communautaire et aux services cliniques en oncologie. Les stratégies de sensibilisation dont la présente étude a montré l'efficacité, c'est-à-dire la publicité marketing (ex. dans les transports en commun), les documents imprimés (ex. affiches) et les publications ou 
vidéos en ligne, peuvent aussi être utiles. Les campagnes de communication narrative sont également un bon outil pour sensibiliser les jeunes femmes et les informer de l'importance de la détection précoce.

Les présentations et les ressources élaborées par l'équipe Shan peuvent être utilisées dans la formation des infirmières en exercice et des étudiants en soins infirmiers pour les informer sur le cancer chez les adolescentes et jeunes adultes. Les chercheurs en soins infirmiers et les infirmières en oncologie travaillant en soins de courte durée sont bien placés pour soutenir les futures recherches qui étudieront l'incidence des retards diagnostiques sur les adolescentes et jeunes adultes. Il importe de rappeler que les adolescentes et jeunes adultes atteints de cancer, mais n'ayant pas encore reçu de diagnostic font tout de même partie du continuum de soins en oncologie.

\section{RÉFÉRENCES}

Bottorff, J. L., Struik, L. L., Bissell, L. J. L., Graham, R., Stevens, J., \& Richardson, C. J. (2014). A social media approach to inform youth about breast cancer and smoking: An exploratory descriptive study. ScienceDirect, 21(2), 159-168. https://doi.org/10.1016/j. colegn.2014.04.002

Canadian Cancer Society [CCS]. (2019). Canadian Cancer Statistics Advisory Committee. Canadian Cancer Statistics 2019. https:// cancer.ca/Canadian-Cancer-Statistics-2019-EN.

Canadian Partnership Against Cancer [CPAC]. (2017). Adolescents \& Young Adults with Cancer: A Systems Performance Report. https://www.partnershipagainstcancer.ca/topics/ adolescents-young-adults-with-cancer/

Canadian Partnership Against Cancer [CPAC]. (2019). Canadian Framework for the Care and Support of Adolescents and Young Adults with Cancer. https://www.partnershipagainstcancer.ca/topics/ framework-adolescents-young-adults /

Chadder, J., \& Coleman, B. (2011). Review of Literature: Risk Factors for Breast Cancer in Young Women Q Health Promotion Campaigns. Team Shan Breast Cancer Awareness for Young Women. https:// teamshan.ca/publications/

Government of Canada. (2018). Tri-Council Policy Statement: Ethical Conduct for Research Involving Humans - TCPS 2. https://ethics. gc.ca/eng/tcps2-eptc2_2018_chapter2-chapitre2.html

Johnson, R. H., Anders, C. K., Litton, J. K., Ruddy, K. J., \& Beyer, A. (2018). Breast cancer in adolescents and young adults. Pediatric Blood a Cancer. 65(12): e27397. https://doi.org/10.1002/pbc.27397.

Kyle, R.G., Forbat, L. \& Hubbard, G. (2012). Cancer awareness among adolescents in Britain: A cross-sectional study. BMC Public Health 12, 580. https://doi.org/10.1186/1471-2458-12-580

Larsen, L., \& Forchuk, C. (2006). Team Shan community project. Evaluation of public awareness campaign materials and breast cancer awareness/knowledge level. https://teamshan.ca/publications/

Larsen, L., \& Forchuk. C. (2008). Team Shan community project. Evaluation of awareness campaign targeting young women. Team Shan Breast Cancer Awareness for Young Women. https:// teamshan.ca/publications/

Larsen, L., \& Forchuk C. (2011). Team Shan Breast Cancer Awareness and Education Project (Calgary). Evaluation of awareness campaign targeting young women. Team Shan Breast Cancer Awareness for Young Women. https://teamshan.ca/publications/

Larsen, L., \& Forchuk, C. (2012). Team Shan Alberta \& Saskatchewan Breast Cancer Awareness for Young Women Project (Year One). Evaluation of awareness campaigns targeting young women. Team Shan Breast Cancer Awareness for Young Women. https:// teamshan.ca/publications/

\section{CONCLUSION}

Les jeunes femmes sont à risque de souffrir de cancer du sein; elles ont donc besoin d'y être sensibilisées et d'être informées sur leur risque de développer la maladie. En connaissant les symptômes du cancer du sein et les stratégies d'autosoins, elles pourront pratiquer l'autodétection et obtenir un diagnostic médical plus rapidement. Grâce à sa stratégie efficace de promotion de la santé, l'équipe Shan est parvenue à rejoindre des milliers de jeunes femmes dans des campus postsecondaires canadiens. Les résultats de la présente étude viennent appuyer l'expansion des stratégies de promotion de la santé afin de sensibiliser les jeunes femmes au risque du cancer du sein et de les informer sur la santé mammaire.

Larsen, L., \& Forchuk, C. (2013). Team Shan Manitoba \& Saskatchewan (Year One) and Alberta Q Saskatchewan (Year Two) Breast Cancer Awareness for Young Women Projects. Evaluation of awareness campaigns targeting young women. Team Shan Breast Cancer Awareness for Young Women. https://teamshan.ca/publications/

Larsen, L., \& Forchuk, C. (2014). Team Shan Manitoba Q Saskatchewan (Year Two) Breast Cancer Awareness for Young Women Project. Evaluation of awareness campaigns targeting young women. Team Shan Breast Cancer Awareness for Young Women. https:// teamshan.ca/publications/

Larsen, L., \& Forchuk, C. (2016). Team Shan UBC Vancouver Breast Cancer Awareness Project. Evaluation of awareness campaign targeting young women. Team Shan Breast Cancer Awareness for Young Women. https://teamshan.ca/publications/

Miles, A. (2017). The psychological implications of diagnostic delay in colorectal cancer patients. Timely diagnosis of symptomatic cancer: Colorectal Cancer. Springer, 103-119. ISBN 9783319652856

Miroshnychenko, A., Rae, C., Tsangaris, E., Breakey, V.R., D’Agostino, N., Klassen, A.F. (2021). Clinical and demographic factors associated with distress in adolescent and young adults with cancer. J Adolesc Young Adult Oncol. https://doi.org/10.1089/ jayao.2020.0196. Epub ahead of print. PMID: 33844943.

Ramphal, R., Aubin, S., Czaykowski, P., De Pauw, S., Johnson, A., McKillop, S., Szwajcer, D., Wilkins, K., \& Rogers, P. (2016). Adolescent and young adult cancer: Principles of care. Current oncology, 23(3), 204-209. https://doi.org/10.3747/co.23.3013

Suffel, S., \& Coleman, B. (2006). Team Shan Community Project literature review. Breast cancer in young women. Team Shan Breast Cancer Awareness for Young Women. https://teamshan.ca/ publications/

Suffel, S., \& Coleman, B. (2007). Team Shan Community Project literature review. Breast cancer in young women. Team Shan Breast Cancer Awareness for Young Women. https://teamshan.ca/ publications/

Symonds, K., Tiseo, L., \& Coleman, B. (2015). Review of the lterature of risk factors for breast cancer in adolescent and young adult women (15-39 years of age) Q health promotion campaigns. Team Shan Breast Cancer Awareness for Young Women. https://teamshan.ca/ publications/ 